\title{
Degradation study on ternary zinc magnesium phosphate glasses.
}

\begin{abstract}
Glasses with composition $(\mathrm{ZnO}) 30(\mathrm{MgO}) \times(\mathrm{P} 2 \mathrm{O} 5) 70-\mathrm{x}(\mathrm{x}=5,8,10,13,15,18$, and 20 mol.\%) have been successfully prepared by the melt-quenching technique. Degradation study has been carried out by means of measuring their chemical durability against buffer solutions with initial $\mathrm{pH}$ values of 4.01, 7.00, and 10.01 at an ambient temperature for up to 30 days. The dissolution rate (D R) was obtained by calculating the measured weight loss of the glasses per unit surface area per unit immersion time. The results show that the glasses have better corrosion resistance in basic solution. It was also found that the weight loss is related to the $\mathrm{MgO}$ concentration with lower $\mathrm{P} 2 \mathrm{O} 5$ concentration exhibiting greater corrosion resistance irrespective of acidic, neutral, or basic solutions as immersion liquid. All the sample surfaces and edges were corroded and the solutions experienced a decrease in $\mathrm{pH}$ values during the duration of the corrosion test.
\end{abstract}

Keyword: Phosphate glasses; Degradation. 